\title{
Rodbot: A Rolling Microrobot for Micromanipulation
}

\section{Conference Paper}

Author(s):

Pieters, Roel S.; Tung, Hsi-Wen; Charreyron, Samuel (D); Sargent, David F.; Nelson, Bradley J.

Publication date:

2015

Permanent link:

https://doi.org/10.3929/ethz-b-000104568

\section{Rights / license:}

In Copyright - Non-Commercial Use Permitted

Originally published in:

https://doi.org/10.1109//CRA.2015.7139764

\section{Funding acknowledgement:}

268004 - Microrobotics and Nanomedicine (EC) 


\title{
RodBot: a Rolling Microrobot for Micromanipulation
}

\author{
Roel Pieters, Hsi-Wen Tung, Samuel Charreyron, David F. Sargent and Bradley J. Nelson
}

\begin{abstract}
We introduce the modelling and control of a rolling microrobot. The microrobot is capable of manipulating micro-objects through the use of a magnetic visual control system. This system consists of a rod-shaped microrobot, a magnetic actuation system and a visual control system. Motion of the rolling microrobot on a supporting surface is induced by a rotating magnetic field. As the robot is submerged in a liquid this motion creates a rising flow in front, a sinking flow behind, and a vortex above the robot, thus enabling non-contact transportation of micro-objects. Besides this fluidvortex approach, the microrobot is also able to manipulate micro-objects via a pushing strategy. We present the design and modelling of the $50 \times 60 \times 300 \mu \mathrm{m}$ micro-agent, the visual control system, and an experimental analysis of the micromanipulation and control methods.
\end{abstract}

\section{INTRODUCTION}

Object manipulation is one of the primary challenges for microrobots operating at the micro-scale as the relative importance of physical effects changes as compared to the macro-scale. Surface effects such as electrostatics dominate over volumetric effects such as weight and inertia, and the fluid mechanics at low Reynolds number is dominated by viscous forces [1]. Actuation principles for microrobotic devices range from electrostatic [2], optical [3] and fluidic techniques [4], [5] to electromagnetic approaches [6]. Magnetic fields as a means of actuation at the microscale is a relatively common and well-understood approach. Field sources can be classified as movable permanent magnets [7], magnetic resonant imaging (MRI) [8] and electromagnetic coils [6], and methods of magnetic energy transfer to the microrobot can be effected by magnetic field gradients, rotating magnetic fields [4] or magnetic resonant frequency actuation [9].

The advantage that fluidic trapping has over other techniques is the non-contact manner of manipulation. This has been shown in [4] for the manipulation of a polystyrene microsphere by a rotating nickel nanowire and in [5] for the manipulation of micro-objects by a micro-manipulator. In particular, Ye et al. [5] show a system of untethered magnetic micro-manipulators that locally induce a rotational fluid flow. The vortex created by the microrobot pulls the object alongside and around the microrobot offering coarse control of object motion.

The control of such microrobotic devises is an active topic of research, please refer to [10] for a survey on planning and control of such automated micromanipulation operations.

Roel Pieters, Samuel Charreyron and Bradley J. Nelson are with the Institute of Robotics and Intelligent Systems, ETH Zurich, Tannenstrasse 3, Zurich, 8092, Switzerland. David F. Sargent is with the Institute of Molecular Biology and Biophysics, ETH Zurich, Schafmattstrasse 20, Zurich, 8093, Switzerland. (e-mail: rpieters@ethz.ch, samuelch@ethz.ch, sargent@mol.biol.ethz.ch, bnelson@ethz.ch).
Particular examples can be found in e.g. [11], which shows the control of multiple microrobots in 2-D space, and in [12], which discusses the control design of a magnetically guided microrobot in blood vessels.

The microrobot discussed here was introduced in [24] and [27]. In this work, we propose the modelling and control of the RodBot, and show additional capabilities such as pushing manipulation. We model and analyse the forces acting on the RodBot as well as on objects indicating the capabilities of the robotic system and the range of objects suitable for micromanipulation.

A complete analysis of the forces and torques acting on the RodBot is presented in Section II. This includes the volumetric, Van der Waals and drag force on the RodBot, as well as the drag torque, and is compared to the magnetic force and torque generated by the magnetic manipulation system. For visual feedback control, the RodBot can be modelled as a standard unicycle mobile robot. A kinematic controller is designed as well as a trajectory generation technique that takes the non-holonomic constraint of the robot into account (Section III). The evaluation of the microrobot, the magnetic manipulation system and the visual control system is discussed in Section IV. Section V presents the conclusions.

\section{DESIGN AND Modelling}

\section{A. The RodBot}

The RodBot (see Fig. 2), is a wireless mobile device with a typical cross section of $50 \times 60 \mu \mathrm{m}$ and a length of 300 to $600 \mu \mathrm{m}$. It is designed as a rectangular polymer (SU-8) rod with internal, transverse soft-magnetic posts consisting of a cobalt-nickel (CoNi) alloy that align with an external magnetic field. When the field direction changes, the RodBot realigns with it. When placed in a rotating magnetic field the RodBot rolls continuously on the supporting surface and generates flow to lift up and trap objects in a vortex above it. Objects ranging from a few microns to several hundreds
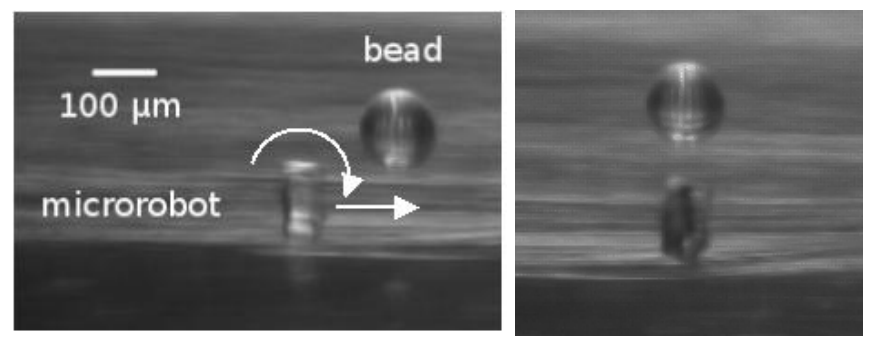

Fig. 1. Sideview of the rolling microrobot lifting up (left) and trapping (right) a polystyrene bead in the vortex. 


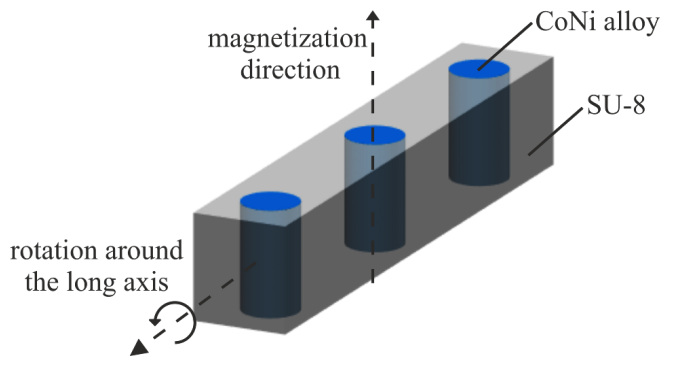

Fig. 2. The RodBot has a polymer body and soft-magnetic posts that align with an external magnetic field.

of microns in size can be lifted and transported to a specific location.

The volumetric vertical force of the micorobot is the combined action of the weight of the robot and its buoyancy

$$
F_{n}=V\left(\rho-\rho_{f}\right) g
$$

where $\rho=r_{m} \rho_{m}+\left(1-r_{m}\right) \rho_{n}, r_{m}=\frac{V_{m}}{V}$, the volume ratio of magnetic to non-magnetic material in the robot, $\rho_{m}$ and $\rho_{n}$ are the densities of the magnetic and non-magnetic materials respectively, $\rho_{f}$ is the density of the surrounding fluid and $g$ is earth's gravitational constant.

\section{B. Van der Waals Force on the RodBot}

The Van der Waals force is considered a long-range adhesive force acting between $0.2-20 \mathrm{~nm}$. Between two flat surfaces it can be described, per unit area, as [13]

$$
F_{v d w} / A_{s}=-\frac{A_{h}}{6 \pi d_{0}^{3}},
$$

with area $A_{s}$ and $d_{0}$ the distance between the surfaces, and $A_{h}$ the Hamaker constant. The minus sign indicates that the force is attractive.

The Van der Waals force decays rapidly with distance and only acts when the microrobot is static on the surface. The distance between the static robot and the surface $d_{0}$ depends on the surface roughness and flatness of the microrobot and the substrate. When in motion, $d_{0} \gg 20 \mathrm{~nm}$ due to the rotation of the microrobot around its long axis which induces a lifting effect on the RodBot. This effect is dependent on the viscosity of the fluid and the angular velocity $\omega$ of the RodBot. For higher values of $\omega>10 \mathrm{rad} / \mathrm{s}, d_{0}$ remains roughly constant (see Fig. 3).

\section{Drag Force and Torque on the RodBot}

As the RodBot translates and rotates, it experiences a drag force and a drag torque. Both the drag force and drag torque acting on the microrobot can be estimated by assuming that the robot is a solid circular cylinder in a slow, low Reynolds number flow perpendicular to its long axis. Analytical models for the drag force and torque acting on a cylinder in open flow can be found in [14] and [15], respectively. In the case of the RodBot, motion is executed in a confined space close to a wall. Per unit length $L$ the drag force is then defined as [16]

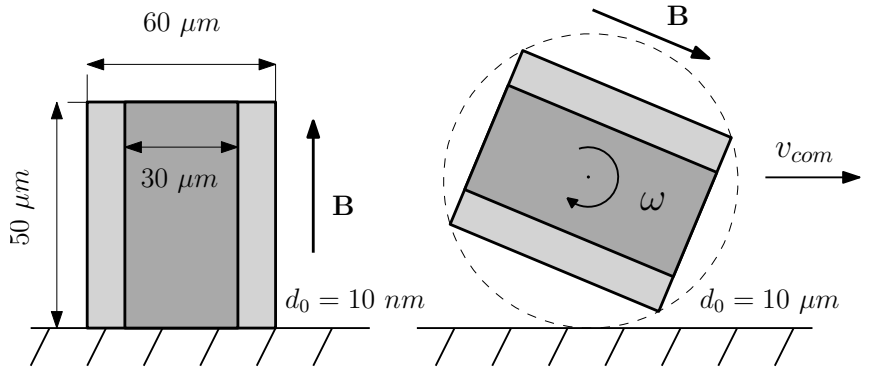

Fig. 3. Sideview of a static RodBot on the surface (left) and a moving RodBot in a liquid (right). The dark grey area depicts $\mathrm{CoNi}$, the light grey area depicts SU-8. $d_{0}$ depicts the gap distance between RodBot and surface. $\mathbf{B}$ and the arrow represent the general magnetic field direction to which the RodBot aligns.

$$
F_{d} / L=\frac{4 \pi \mu_{d} U}{\log \frac{2 d_{c}}{r_{c}}-1 / 4\left(\frac{r_{c}}{d_{0}}\right)^{2}},
$$

where $\mu_{d}$ is the fluid's dynamic viscosity, $U$ is the velocity of the fluid, which is equal to the velocity of the microrobot $U=v_{c o m}$, and $L$ is the length of the cylinder. The parameters corresponding to the wall interaction are $r_{c}$, the radius of the cylinder, and $d_{c}$, the distance between the centre of the microrobot and the wall.

The drag torque per unit length $L$ of a cylinder rotating close to a wall is given by [17]

$$
\tau_{d} / L=\frac{4 \pi \mu_{d} \omega r_{c}^{2}}{\sqrt{1-k^{2}}},
$$

where $k=r_{c} / d_{c}$, and $\omega$ is the rotational velocity of the cylinder.

\section{Drag Force on Objects}

The viscous drag force is the major force acting to overcome gravity and stiction and lifts objects for transport. This drag force results from the motion of the RodBot and can be determined from Stokes' law. The drag force experienced by a spherical object (e.g., a protein crystal) due to a low Reynolds number fluid flow can be described by [13]

$$
F_{d s} \simeq 6 \pi \mu_{d} r_{o} U
$$

where $\mu_{d}$ is the dynamic viscosity of the fluid, $r_{o}$ is the radius of the sphere, and $U$ is the velocity of the sphere relative to the fluid. When the object is close to a wall equation (5) is corrected as [18]

$$
F_{d s}^{*}=F_{d s}\left(1+\frac{9}{16} \frac{r_{o}}{d_{c}}\right)^{-1}
$$

Although objects can be nonspherical, the drag force experienced can, nevertheless, be estimated by equation (5) and (6). 


\section{E. Magnetic Manipulation System}

The microrobot is exposed to an externally applied magnetic field $\mathbf{H}$ with a flux density $\mathbf{B}=\mu_{0} \mathbf{H}$, where $\mu_{0}$ is the permeability of vacuum. The torque that acts on the microrobot can then be defined as

$$
\tau=V \mathbf{M} \times \mathbf{B}
$$

where $\mathbf{M}$ is the volume magnetization in $[A / m]$ of the object of volume $V$.

The force acting on the microrobot is described as

$$
\mathbf{F}=V(\mathbf{M} \cdot \nabla) \mathbf{B} .
$$

Assuming no electric current is flowing through the microrobot, Maxwell's equations require that $\nabla \times \mathbf{B}=\mathbf{0}$, from which the force can be expressed as

$$
\mathbf{F}=V\left[\frac{\partial \mathbf{B}}{\partial x} \frac{\partial \mathbf{B}}{\partial y} \frac{\partial \mathbf{B}}{\partial z}\right]^{T} \mathbf{M}
$$

The magnetic field throughout the workspace of a set of static electromagnets can be computed at point $\mathbf{P}$ for any given electromagnet $e$. The magnetic field due to this electromagnet can be expressed as the vector $\mathbf{B}_{e}(\mathbf{P})$, whose magnitude varies linearly with the current through the electromagnet. When assuming an ideal soft-magnetic material with negligible hysteresis and keeping the cores within their linear magnetization regions, the field contributions of the individual currents superimpose linearly. This can be expressed as the $3 \times n$ unit-field contribution matrix $\mathcal{B}(\mathbf{P})$

$$
\mathbf{B}(\mathbf{P})=\left[\tilde{\mathbf{B}}_{1}(\mathbf{P}) \ldots \tilde{\mathbf{B}}_{n}(\mathbf{P})\right]\left[\begin{array}{c}
i_{1} \\
\vdots \\
i_{n}
\end{array}\right]=\mathcal{B}(\mathbf{P}) I,
$$

where vector I holds the currents in each coil.

The magnetic potential energy $E_{p}$ due to the torque acting on the RodBot defined by (7) and of magnitude $\tau=V|M||B| \sin \theta$ depends upon its orientation with respect to the magnetic field and is highest when it is perpendicular to the magnetic field and lowest when aligned

$$
E_{p}=-V \mathbf{M} \cdot \mathbf{B}
$$

The potential energy is converted to kinetic energy $E_{k}$ by the microrobot and results in the forward and angular velocity of the device

$$
E_{k}=\frac{1}{2} m_{r} v_{c o m}^{2}+\frac{1}{2} I \omega^{2}
$$

where $v_{c o m}$ is the forward velocity of the center of mass and $\omega$ is the angular velocity. The moment of inertia of the robot can be modelled as a cylinder $I=m_{r} r_{c}^{2} / 2$, where $m_{r}$ and $r_{c}$ are the mass and radius of the cylinder, respectively. Due to the slip of the robot $v_{\text {com }}<r_{c} \omega$, which can be written as $v_{\text {com }}=\mu_{s} r_{c} \omega$, where $0<\mu_{s} \leq 1$ is the slip coefficient, which depends on the viscosity of the liquid as well as the characteristics of the surfaces of the RodBot and the substrate.

\section{Visual Control System}

Due to the design of the robot and the method of actuation (i.e., a rotating magnetic field induces a forward velocity), the system is limited to non-holonomic motion, corresponding to 'classical' unicycle mobile robots. Experimentally, a top camera observes the scene and image processing detects the position and orientation of the RodBot and provides feedback for the visual control system.

\section{A. Kinematic modelling}

As the RodBot can translate and rotate on a plane, its configuration space is represented by $\mathbf{S E}(2)=\mathbb{R}^{2} \times \mathbf{S 0}(2)$, and the configuration vector $\mathbf{q}=[\mathbf{x}, \mathbf{y}, \theta]^{\mathbf{T}}$. The kinematics can be approximated with the standard unicycle-model as

$$
\left\{\begin{array}{l}
\dot{x}=v \cos \theta_{m} \\
\dot{y}=v \sin \theta_{m} \\
\dot{\theta}_{c}=\omega_{c} .
\end{array}\right.
$$

The degrees of freedom to be controlled are the forward velocity of the robot, $v$, and the steering velocity of the robot, $\omega_{c}$. As depicted in Fig. 4 , let $P$ be a virtual target on the trajectory and $Q$ be the center of mass of the RodBot. With respect to an inertial reference frame $\{I\}, Q$ and $P$ are denoted as $Q_{\{I\}}=\left[q_{x}, q_{y}, 0\right]^{T}$ and $P_{\{I\}}=\left[p_{x}, p_{y}, 0\right]^{T}$. A Cartesian error between the RodBot and the trajectory is then defined as $\mathbf{e}(\mathbf{q})=P-Q$.

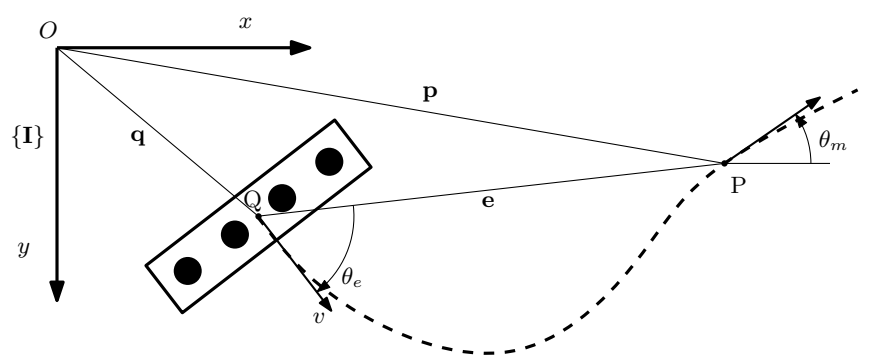

Fig. 4. $Q$ denotes the center of the RodBot, $P$ denotes a virtual target on the trajectory. The controller is designed to regulate $\mathbf{e}=P-Q$ to zero.

\section{B. Kinematic controller}

The path- or trajectory-following problem for unicycles or wheeled mobile robots is typically solved using model-based control laws [19], [20]. We propose a model-free approach that assumes that the generated path or trajectory handles the non-holonomic and kinematic constraints of the RodBot. The controller is defined as

$$
\left\{\begin{array}{l}
v=v_{\max } \tanh \left(k_{v}\|\mathbf{e}\|\right), \\
\theta_{m}=\arctan \left(\frac{e_{y}}{e_{x}}\right), \\
\ddot{s}=k_{d}\left(\|\mathbf{e}\|-d_{a}\right),
\end{array}\right.
$$

where $v_{\max }$ defines a maximum forward velocity and $k_{v}$ and $k_{d}$ are control gains. An artificial third input $\ddot{s}$ is added which controls the acceleration of the virtual target $P$ on the trajectory. It is defined as the difference between $d_{a}$, a desired distance between the virtual target and the RodBot, 
and the actual difference $\|\mathbf{e}\|$ as measured by the tracker. The controller attempts to maintain a constant distance between the virtual target and the RodBot.

\section{Motion Planning}

As mentioned in section III-B, the non-holonomic and kinematic constraints of the RodBot are handled by the motion planner, which generates a planar trajectory with two polynomial functions. A trajectory of order $m$ is defined as:

$$
q(t)=a_{0}+a_{1} t+a_{2} t^{2}+\cdots+a_{m} t^{m},
$$

with $t \in\left[t_{i}, t_{f}\right]$, where $t_{i}$ indicates the initial time instant $(t=0)$ and $t_{f}$ indicates the final time instant. A general solution is acquired by solving a system of linear equations:

$$
\mathbf{T a}=\mathbf{q}_{c},
$$

where $\mathbf{T}$ is the Vandermonde matrix [21]. The unknown polynomial coefficients are $\mathbf{a}=\left[a_{0}, a_{1}, \ldots, a_{m}\right]^{T}$, and $\mathbf{q}_{c}$ lists the $(m+1)$ constraints that the polynomial should satisfy. The coefficients in a can be computed as

$$
\mathbf{a}=\mathbf{T}^{\dagger} \mathbf{q}_{c}
$$

where $\mathbf{T}^{\dagger}$ represents the pseudo-inverse of $\mathbf{T}$.

\section{EVALUATION}

The magnetic control system is described in [23] and includes the eight-coil Magnetic Field Generator (MFG), a microscope with camera for visual feedback and a container enclosing the RodBot and the micro-objects (Fig. 5). The entire system is controlled through $\mathrm{C}++$ by a single computer running Ubuntu Linux. The system is capable of 5-DOF wireless control of micro- and nano-structures (3DOF position, 2-DOF pointing orientation) within a spherical workspace with a diameter of approximately $10 \mathrm{~mm}$. The MFG can generate magnetic fields and field gradients up to $50 \mathrm{mT}$ and $5 \mathrm{~T} / \mathrm{m}$ at frequencies up to $2 \mathrm{kHz}$. For the fabrication of the RodBot, please refer to [24].

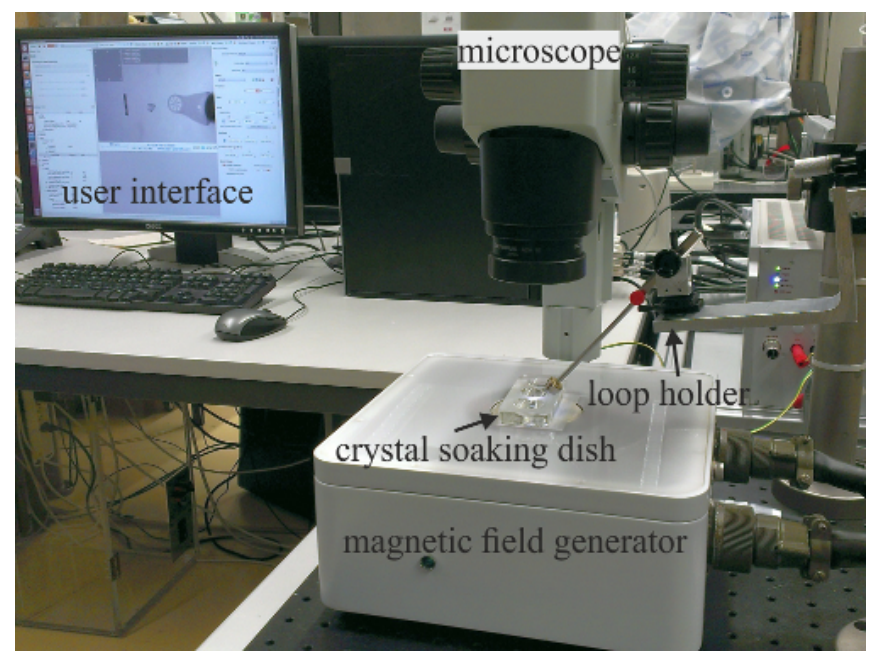

Fig. 5. The experimental system contains the eight-coil magnetic field generator, a microscope with camera for visual feedback, and a desktop PC for processing [27].

\section{A. Forces, Torques and Magnetic Actuation}

The torque and force acting on the microrobot due to the magnetic field can be determined via equations (7) and (8). The magnetic properties (magnetization) of the CoNi posts in the RodBot are determined as $M=380 \mathrm{kA} / \mathrm{m}$. With the (moderate) magnetic field parameters as $\nabla B=1 \mathrm{mT} / \mathrm{m}$ and $B=10 \mathrm{mT}$, a typical torque and force equals $\tau_{m}=$ $0.7 n N m$ and $F_{m}=68 n N$.

Table I lists typical force and torque values acting on the RodBot as described in Section II. The parameters used to obtain these measures can be found in Table II. From this it can be concluded that, when in motion, the volumetric vertical force of the microrobot is dominant, and confirms that the magnetic manipulation system can effectively actuate the microrobot.??

TABLE I

FORCE COMPARISON

\begin{tabular}{|l|c|c|c|}
\hline $\mathrm{U}$ & $0 \mathrm{~mm} / \mathrm{s}$ & $0.5 \mathrm{~mm} / \mathrm{s}$ & $1 \mathrm{~mm} / \mathrm{s}$ \\
\hline$F_{n}$ & $17.8 \mathrm{nN}$ & $17.8 \mathrm{nN}$ & $17.8 \mathrm{nN}$ \\
\hline$F_{v d w}$ & $8 \mu \mathrm{N}$ & $<p N$ & $<p N$ \\
\hline$F_{d}$ & 0 & $0.2 \mathrm{nN}$ & $0.4 \mathrm{nN}$ \\
\hline$F_{d s}^{*}$ & 0 & $0.6 \mathrm{nN}$ & $1.2 \mathrm{nN}$ \\
\hline$\tau_{d}$ & 0 & $0.3 \mathrm{pNm}$ & $0.6 \mathrm{pNm}$ \\
\hline$F_{m}$ & $68 \mathrm{nN}$ & $68 \mathrm{nN}$ & $68 \mathrm{nN}$ \\
\hline$\tau_{m}$ & $0.7 \mathrm{nNm}$ & $0.7 \mathrm{nNm}$ & $0.7 \mathrm{nNm}$ \\
\hline
\end{tabular}

TABLE II

PHYSICAL PARAMETERS

\begin{tabular}{|c||c|c|}
\hline parameter & variable & value \\
\hline Density water & $\rho_{w}$ & $1000 \mathrm{~kg} \cdot \mathrm{m}^{-3}$ \\
\hline Density 20\%PEG3350 & $\rho_{P E G}$ & $1050 \mathrm{~kg} \cdot \mathrm{m}^{-3}$ \\
\hline Density SU-8 & $\rho_{S U-8}$ & $1200 \mathrm{~kg} \cdot \mathrm{m}^{-3}$ \\
\hline Density CoNi & $\rho_{C o N i}$ & $8900 \mathrm{~kg} \cdot \mathrm{m}^{-3}$ \\
\hline Vaccuum permeability & $\mu_{0}$ & $4 \pi \times 10^{-7} \mathrm{~T} \cdot \mathrm{m} / \mathrm{A}$ \\
\hline Hamaker constant & $A_{h}$ & $1 e^{-20} \mathrm{~J}$ \\
\hline Gap distance & $d_{0}$ & $10 \mathrm{~nm}-10 \mu \mathrm{m}$ \\
\hline Cylinder radius & $r_{c}$ & $78 \mu \mathrm{m}$ \\
\hline RodBot length & $L$ & $300 \mu \mathrm{m}$ \\
\hline Dynamic viscosity water & $\mu_{d}$ & $1 \mathrm{mPa} \cdot \mathrm{s}$ \\
\hline Dynamic viscosity 20\%PEG3350 & $\mu_{d}$ & $9.3 \mathrm{mPa} \cdot \mathrm{s}$ \\
\hline Kinematic viscosity water & $\nu$ & $1 \mathrm{~mm} \cdot \mathrm{s}$ \\
\hline Sphere radius & $r_{o}$ & $100 \mu \mathrm{m}$ \\
\hline Magnetization CoNi & $M$ & $380 \mathrm{kA} / \mathrm{m}$ \\
\hline
\end{tabular}

\section{B. Object Manipulation}

The fluidic vortex above the RodBot is generated due to the robot's rotation and the fluid's no-slip condition at the boundaries. This means that the shape of the RodBot need not necessarily be a rectangular cylinder to achieve fluidic trapping [26]. The motion of the RodBot creates a rising flow in front of it, a sinking flow behind it, and a vortex above its body [24]. This phenomena is identified and analysed in [17] and [26], but not exploited for transportation utilizing a mobile microrobot. The gentle, fluidic force acting on microobjects is in the range of a few nano-Newtons to hundreds of nano-Newtons (see Section II-D and Table I and II). The RodBot is capable of trapping objects ranging from a few microns to sub-millimeters in major dimensions. This is 
shown in Fig. 8 in which the RodBot lifts, transports, and deposits a $300 \mu \mathrm{m}$ lysozym crystal towards an extraction tool without physical contact. An analysis on the applicability of using the RodBot for crystal harvesting is treated in [27].

Besides lifting objects up into the vortex, the RodBot can also manipulate objects by pushing. This manipulation technique is shown in Fig. 9, where the RodBot assembles four micro-objects (triangles, $200 \times 350 \mu \mathrm{m}$ ) densely packed into a narrow channel (750 $\mu \mathrm{m}$ wide) within two minutes. This assembly task was part of the Mobile Microrobotics Challenge at the 2014 IEEE International Conference on Robotics and Automation (ICRA).

\section{Visual Control Evaluation}

The RodBot is visually identifiable by its dark magnetic posts. These features are found by segmenting the grayscale input image with an adaptive threshold and a subsequent morphological closing step. Simple blob detection then selects candidate features based on their size. In the feature extraction step the relevant features must be selected with heuristic-based filtering. For the RodBot, two visual characteristics are applicable. First, the number of soft-magnetic posts is known a priori. Second, all posts should appear along a straight line and should be evenly spaced. The algorithm starts with a calibration procedure to determine the spacing between magnetic posts $d_{p}$. Thereafter, a detected set of features is given a score penalizing orthogonal distance to the colinear line $d_{\perp}\left(p_{k}\right)$ and transverse distance to the supposed location $d_{\|}\left(p_{k}\right)$. The total score of the consensus set is defined as:

$$
\operatorname{score}(S)=|S|-\sum_{p_{k} \in S} \frac{d_{\perp}\left(p_{k}\right)}{d_{p}^{2}}+2 \frac{d_{\|}\left(p_{k}\right)}{d_{p}},
$$

where $p_{k}$ is the set of $k \in\{1, \ldots, l\}$ candidate features and $|S|$ represents the cardinality of the feature set (see Fig. 6).

A line is fit through all $p_{i}$ candidate features with the predefined feature set size (i.e., the number of posts). The candidate feature set with the best score is kept, and, if this score exceeds a certain threshold, the RodBot is assumed to have been detected correctly. Finally, to account for measurement noise and false or missing measurements an extended Kalman filter (EKF) [25] is used, which includes a linearised model of the RodBot's unicycle kinematics (13).

Fig. 7 shows the RodBot tracking a desired trajectory (solid white line). The blue solid line shows the executed motion of the robot. The deviation between desired and measured trajectory does not exceed $50 \mu \mathrm{m}$.

\section{Conclusions}

We presented the modelling and control of a rolling microrobot, the RodBot. The microrobot is transversely magnetized and can roll around its long axis on a surface, generating a rising flow in front, a sinking flow behind, and a vortex above itself to trap and transport micro-objects (polystyrene beads and protein crystals) ranging from tens to hundreds of micrometers in size. Besides lifting objects via
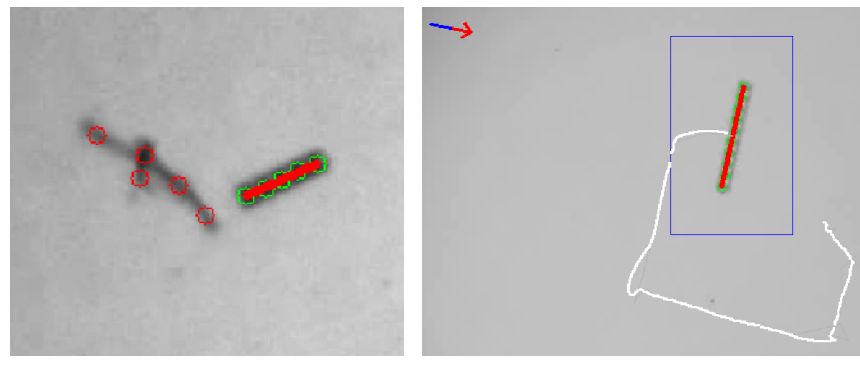

Fig. 6. Successful detection of the RodBot with five posts (left image) and ten posts (right image). The solid red line indicates the detected robot, the green circles the correctly detected underlying features. The left image shows also classified features not belonging to the RodBot (red circles). The right image shows the region of interest for tracking, the orientation of the RodBot (top-left arrow) and the measured (white) positions of the RodBot.

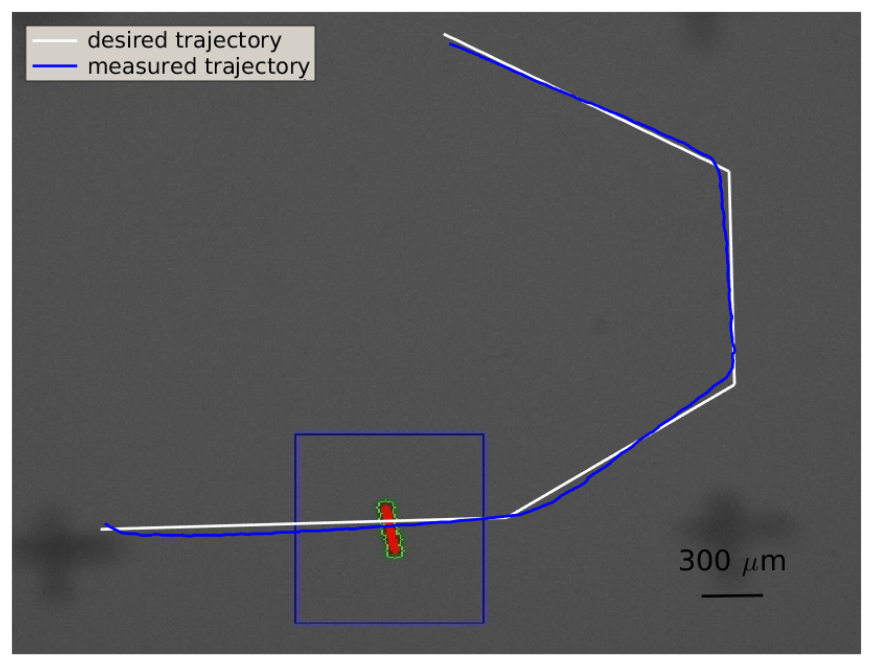

Fig. 7. Tracking control of the RodBot. The deviation between desired and measured trajectory does not exceed $50 \mu \mathrm{m}$.

this vortex, the RodBot can also be used for pushing tasks. We proposed an analysis of the forces and torques acting on the microrobot and on objects due to the environment and due to the magnetic manipulation system. The motion of the robot is controlled with visual feedback and a trajectory to incorporate its kinematic and non-holonomic constraints.

\section{ACKNOWLEDGMENT}

The authors would like to thank Maurice Gonzenbach for work on the detection of the RodBot. This work is funded by the European Research Council Advanced Grant "Microrobotics and Nanomedicine (BOTMED)".

\section{REFERENCES}

[1] J. Abbott, Z. Nagy, F. Beyeler, and B. Nelson, "Robotics in the small, part I: Microbotics,' IEEE Robotics Automation Magazine, vol. 14, no. 2, pp. 92-103, 2007.

[2] C.-J. Kim, A. Pisano, and R. Muller, "Silicon-processed overhanging microgripper," Journal of Microelectromechanical Systems, vol. 1, no. 1 , pp. $31-36,1992$.

[3] A. Ashkin, J. M. Dziedzic, J. E. Bjorkholm, and S. Chu, "Observation of a single-beam gradient force optical trap for dielectric particles," Optical Letters, vol. 11, no. 5, pp. 288-290, 1986. 

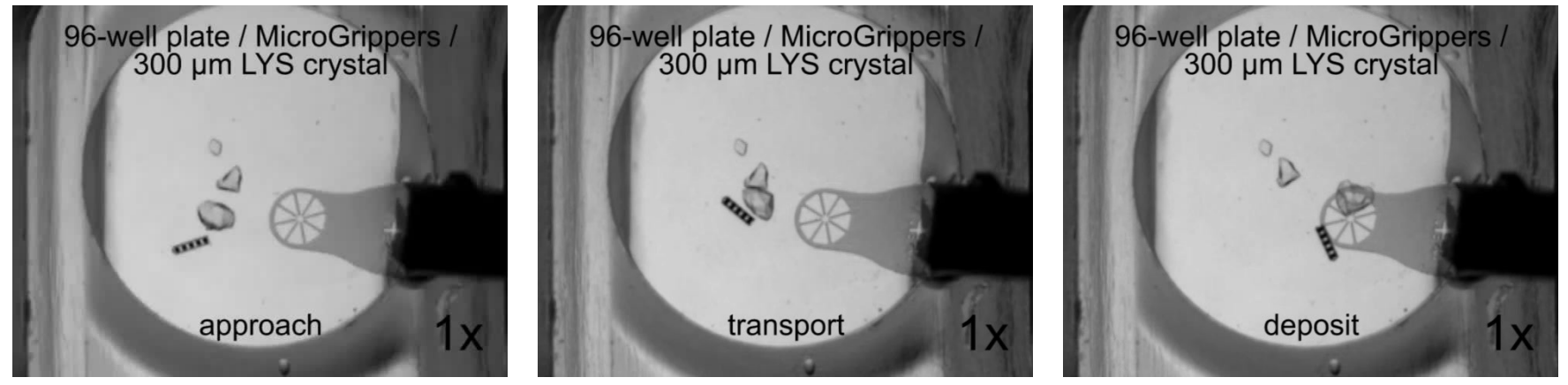

Fig. 8. Microscopic view of manipulation with a fluidic vortex. The RodBot lifts, transports and deposits a lysozym crystal towards an extraction-tool (MicroGripper ${ }^{\mathrm{TM}}$ ) without physical contact. The RodBot and the crystal both have a maximum extent of $300 \mu \mathrm{m}$ and are submerged in a $20 \%$ PEG3350 solution [27].
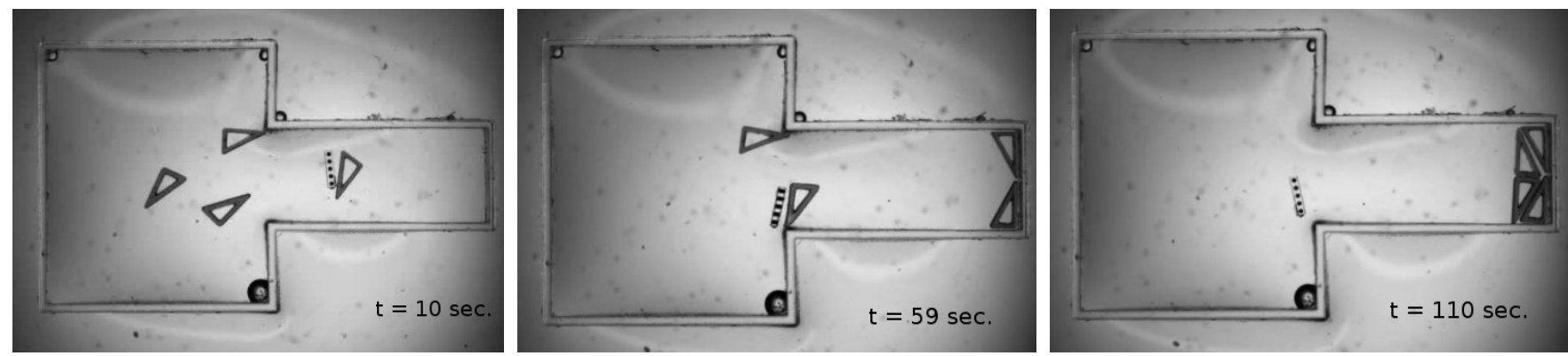

Fig. 9. Microscopic view of manipulation via pushing. The RodBot assembles four micro-objects (triangles, $200 \times 350 \mu m$ ) densely packed into a narrow channel (750 $\mu \mathrm{m}$ wide) within two minutes. This assembly task was part of the Mobile Microrobotics Challenge at the IEEE International Conference on Robotics and Automation (ICRA), 2014. The RodBot, arena, and triangles are submerged in DI water.

[4] L. Zhang, T. Petit, Y. Lu, B. E. Kratochvil, K. E. Peyer, R. Pei, J. Lou, and B. J. Nelson, "Controlled propulsion and cargo transport of rotating nickel nanowires near a patterned solid surface," ACS Nano, vol. 4, no. 10, pp. 6228-6234, 2010.

[5] Z. Ye, E. Diller, and M. Sitti, "Micro-manipulation using rotational fluid flows induced by remote magnetic micro-manipulators," Journal of Applied Physics, vol. 112, no. 6, p. 064912, 2012.

[6] M. Kummer, J. Abbott, B. Kratochvil, R. Borer, A. Sengul, and B. Nelson, "Octomag: An electromagnetic system for 5-dof wireless micromanipulation," IEEE Transactions on Robotics, vol. 26, no. 6, pp. 1006-1017, 2010.

[7] D. Meeker, E. H. Maslen, R. C. Ritter, and F. Creighton, "Optimal realization of arbitrary forces in a magnetic stereotaxis system," IEEE Transactions on Magnetics, vol. 32, no. 2, pp. 320-328, 1996.

[8] S. Martel, M. Mohammadi, O. Felfoul, Z. Lu, and P. Pouponneau, "Flagellated magnetotactic bacteria as controlled mri-trackable propulsion and steering systems for medical nanorobots operating in the human microvasculature.," Int. Journal of Robotics Research, vol. 28, pp. 571-582, 2009.

[9] H.-W. Tung, M. Maffioli, D. Frutiger, K. Sivaraman, S. Pane, and B. Nelson, "Polymer-based wireless resonant magnetic microrobots," IEEE Transactions on Robotics, vol. 30, no. 1, pp. 26-32, 2014.

[10] A. Banerjee and S. Gupta, "Research in automated planning and control for micromanipulation," IEEE Transactions on Automation Science and Engineering, vol. 10, no. 3, pp. 485-495, 2013.

[11] E. Diller, S. Floyd, C. Pawashe, and M. Sitti, "Control of multiple heterogeneous magnetic microrobots in two dimensions on nonspecialized surfaces," IEEE Transactions on Robotics, vol. 28, no. 1, pp. 172-182, 2012.

[12] L. Arcese, M. Fruchard, and A. Ferreira, "Adaptive controller and observer for a magnetic microrobot," IEEE Transactions on Robotics, vol. 29, no. 4, pp. 1060-1067, 2013.

[13] B. Bhushan, Handbook of Micro/Nano Tribology. CRC Press, 1998.

[14] M. M. Tirado and J. G. de la Torre, "Translational friction coefficients of rigid, symmetric top macromolecules. application to circular cylinders," The Journal of Chemical Physics, vol. 71, no. 6, 1979.

[15] M. M. Tirado and J. G. de la Torre, "Rotational dynamics of rigid, symmetric top macromolecules. application to circular cylinders," The Journal of Chemical Physics, vol. 73, no. 4, pp. 1986-1993, 1980.

[16] Y. Takaisi, "Note on the drag on a circular cylinder moving with low speeds in a semi-infinite viscous liquid bounded by a plane wall," $J$. of the Physical Society of Japan, vol. 11, no. 9, pp. 1004-1008, 1956

[17] S. Champmartin, A. Ambari, and N. Roussel, "Flow around a confined rotating cylinder at small reynolds number," Physics of Fluids (1994present), vol. 19, no. 10, 2007.

[18] F. Charru, E. Larrieu, J.-B. Dupont, and R. Zenit, "Motion of a particle near a rough wall in a viscous shear flow," Journal of Fluid Mechanics, vol. 570, pp. 431-453, 2007.

[19] Z.-P. Jiang, E. Lefeber, and H. Nijmeijer, "Saturated stabilization and tracking of a nonholonomic mobile robot," Systems \& Control Letters, vol. 42 , no. 5 , pp. 327 - 332, 2001.

[20] D. Soetanto, L. Lapierre, and A. Pascoal, "Adaptive, non-singular pathfollowing control of dynamic wheeled robots," in Proc. of 42nd IEEE Conference on Decision and Control, vol. 2, pp. 1765-1770, 2003.

[21] L. Biagiotti and C. Melchiorri, Trajectory Planning for Automatic Machines and Robots. Springer Berlin Heidelberg, 2008.

[22] T. Flash and N. Hogan, "The coordination of arm movements: an experimentally confirmed mathematical model," Journal of Neuroscience, vol. 5, no. 7, pp. 1688-1703, 1985.

[23] S. Schuerle, S. Erni, M. Flink, B. Kratochvil, and B. Nelson, "Threedimensional magnetic manipulation of micro- and nanostructures for applications in life sciences," IEEE Transactions on Magnetics, vol. 49, no. 1, pp. 321-330, 2013.

[24] H.-W. Tung, K. E. Peyer, D. F. Sargent, and B. J. Nelson, "Noncontact manipulation using a transversely magnetized rolling robot," Applied Physics Letters, vol. 103, p. 114101, September 2013.

[25] S. Julier and J. Uhlmann, "Unscented filtering and nonlinear estimation," Proceedings of the IEEE, vol. 92, no. 3, pp. 401-422, 2004.

[26] M. Hellou and M. Coutanceau, "Cellular stokes flow induced by rotation of a cylinder in a closed channel," Journal of Fluid Mechanics, no. 236 , pp. 557-577, 1992.

[27] H.-W. Tung, D. F. Sargent, and B. J. Nelson, "Protein crystal harvesting using the rodbot - a wireless, mobile microrobot," Journal of Applied Crystallography, vol. 4, pp. 692-700, 2014. 